\section{Normas y estándares aplicables a los campos electromagnéticos de radiofrecuencias en América Latina: guía para los límites de exposición y los protocolos de medición}

\author{
Jorge Skvarca' y Aníbal Aguirre ${ }^{2}$
}

Forma de citar: Skvarca J, Aguirre A. Normas y estándares aplicables a los campos electromagnéticos de radiofrecuencias en América Latina: guía para los límites de exposición y los protocolos de medición. Rev Panam Salud Publica. 2006;20(2/3):205-12.

Palabras clave: normas de calidad ambiental, campos electromagnéticos, exposición a la radiación.

\footnotetext{
1 Departamento de Electrónica, Facultad de Ingeniería de la Universidad de Buenos Aires, Buenos Aires, Argentina. La correspondencia se debe dirigir a: Jorge Skvarca, Moreno No. 1652, Buenos Aires, CP 1093, Argentina. Correo electrónico: jorgesk@hotmail.com

2 Cátedra de Sistemas Inalámbricos, Facultad de Ingeniería de la Universidad de Buenos Aires, Buenos Aires, Argentina.
}

SINOPSIS

Las nuevas tecnologías que utilizan campos electromagnéticos (CEM) ofrecen a la humanidad inmensos beneficios. Además de emplearse en la transmisión de energía eléctrica, las telecomunicaciones y en equipos industriales, médicos y electrodomésticos, los CEM tienen otras muchas y muy diversas aplicaciones. Algunos estudios demuestran que la exposición a los CEM podría producir efectos adversos a la salud, como cáncer y cambios en el comportamiento de las personas. Aunque hasta el presente no se ha demostrado que la exposición a CEM de baja intensidad provoque estos efectos, se trabaja por lograr un consenso científico al respecto y por establecer normas de seguridad adecuadas. La responsabilidad de desarrollar y promover los estándares de seguridad ha recaído fundamentalmente en organizaciones y agencias especializadas reconocidas internacionalmente; sin embargo, las autoridades sanitarias nacionales deben participar activamente en ese proceso. La Organización Panamericana de la Salud ha decidido promover investigaciones científicas y epidemiológicas con vistas a proponer guías y estándares armonizados. Algunos países de América Latina, como Argentina, Bolivia, Brasil, Chile, Colombia, Costa Rica, Ecuador, México, Perú y Venezuela ya cuentan con leyes específicas, aunque generalmente parciales o incompletas, basadas en las recomendaciones internacionales. En este artículo se describen las normas establecidas en América Latina y se analizan los diferentes enfoques de cada una de ellas.

Los problemas asociados con el uso de las radiaciones no ionizantes han cobrado importancia a medida que aumenta la utilización de las fuentes que las generan. Entre estas fuentes se encuentran las líneas de transmisión de energía eléctrica (1) y las estaciones de transformación que generan campos de muy baja frecuencia $(50-60 \mathrm{~Hz})$, las fuentes de alimentación conmutadas, las estaciones de radiodifusión de amplitud modulada (525-1 735 $\mathrm{kHz}$ ), las estaciones de radiodifusión de frecuencia modulada (88-108 MHz), las estaciones de televisión "por aire" en las bandas de frecuencias muy altas (VHF) y ultraaltas (UHF) y los sistemas de comunicación móviles por celdas (800 y 1900 MHz), la soldadura por radiofrecuencia de uso industrial, los equipos de tecnología médica que utilizan radiaciones de radiofrecuencias y los rayos láser y del espectro ultravioleta cercano en sus diversas aplicaciones clínicas, entre otros (figura 1). Los dispositivos de mantenimiento de energía eléctrica que al cargarse generan grandes campos estáticos, los sistemas de resonancia magnética nuclear y los ferro- 
FIGURA 1. Espectro electromagnético de las radiaciones ionizantes

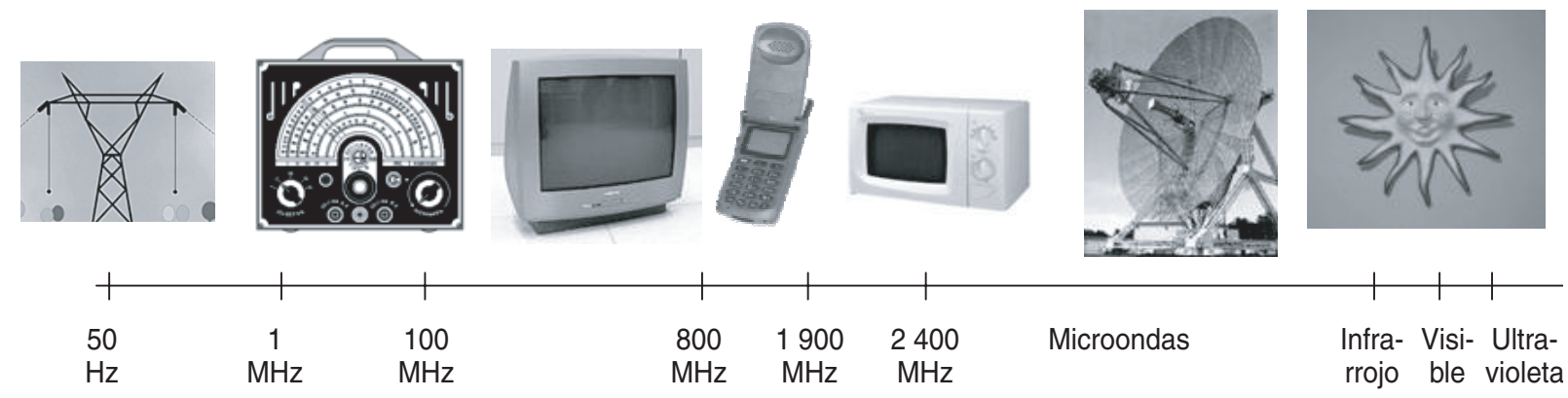

carriles eléctricos por corriente continua (de $0 \mathrm{~Hz}$ ), si bien no se propagan por ser estáticos, también son objeto de estudio de los especialistas en radiaciones no ionizantes.

Los campos electromagnéticos (CEM) generados por las mencionadas fuentes en los grandes centros urbanos han dado origen a las disciplinas que estudian la acción de las radiaciones no ionizantes sobre las personas, así como la compatibilidad y la interferencia electromagnéticas con equipos y sistemas electrónicos o electromecánicos.

En el presente artículo se examina la problemática que plantea el uso de las radiaciones no ionizantes en América Latina y el Caribe, se analizan las normas establecidas para proteger a la población contra estas radiaciones y sus fundamentos y se expone la legislación al respecto adoptada por algunos países latinoamericanos.

\section{LAS RADIACIONES NO IONIZANTES}

Para este análisis se tomaron en cuenta solo las fuentes emisoras de radiaciones no ionizantes destinadas a los servicios de comunicaciones (de 30 $\mathrm{kHz}$ a $300 \mathrm{GHz}$ ). Se debe destacar que las radiaciones provenientes de fuentes de menor frecuencia merecen igual atención; sin embargo, el análisis detallado de sus particularidades excede el alcance del presente trabajo.

\section{Magnitudes y unidades de campos electromagnéticos}

Los CEM de radiofrecuencias y microondas (de $0,3 \mathrm{GHz}$ a $300 \mathrm{GHz}$ ) se caracterizan mediante la intensidad del campo eléctrico (E), expresada en voltios por metro $(\mathrm{V} / \mathrm{m})$, y la intensidad del campo magnético $(\mathrm{H})$, medida en amperios por metro $(\mathrm{A} / \mathrm{m})$ o en teslas $(\mathrm{T})$. El flujo de energía de la onda electromagnética, conocido como densidad de po- tencia, se propaga perpendicularmente a estos dos componentes y se cuantifica mediante un parámetro conocido como vector de Poynting (S). La longitud de onda $(\lambda)$ de la propagación y su frecuencia (f) son dos características relacionadas entre sí por la velocidad de propagación de las ondas electromagnéticas (c), que es igual a la velocidad de la luz en el vacío (300 $000 \mathrm{~km} / \mathrm{s})$.

Otro aspecto importante relacionado con los efectos biológicos de las radiaciones no ionizantes provocados por la interacción de los campos de radiofrecuencias con sistemas biológicos es la tasa de absorción específica, que está dada por la energía absorbida por unidad de tiempo (potencia) expresada en vatios $(\mathrm{W})$ por unidad de masa corporal en kilogramos $(\mathrm{W} / \mathrm{kg})$. La tasa de absorción específica es la unidad dosimétrica empleada para cuantificar los efectos biológicos y definir los límites de exposición.

\section{La necesidad de una norma}

Si en un punto urbano se detecta un CEM proveniente de una emisora de radio de frecuencia modulada y de una radiocelda de telefonía móvil cercana, cabe preguntarse: ¿Cuál es el nivel de CEM permitido? ¿Qué diferencia hay entre el CEM proveniente de la estación de frecuencia modulada y el proveniente de la radiocelda? ¿La radiocelda y la estación de frecuencia modulada están situadas en lugares adecuados? ¿Cómo se deben medir los CEM para poder caracterizarlos correctamente?

Para responder a estas preguntas se deben tener en cuenta las normas que establecen las condiciones y parámetros fundamentales para el trabajo con radiaciones no ionizantes (2), entre ellos:

- los valores de exposición máxima permitida para un espectro de frecuencias dado;

- los lugares donde se pueden emplazar los sistemas que emiten radiaciones y las condiciones que deben cumplir; 
- los procedimientos (protocolos de medición) que deben emplearse para caracterizar los CEM, a fin de obtener valores confiables que puedan compararse con las normas que establecen los niveles de exposición máxima permitida.

La elaboración de estas normas corresponde a diferentes ramas institucionales; sin embargo, establecer los valores máximos permitidos de exposición es competencia de las autoridades sanitarias (nacionales e internacionales), especialmente de las dedicadas a la aplicación de técnicas vinculadas con la radiofísica y la biocompatibilidad electromagnética (3). No obstante, la elaboración de las normas que regulan los procedimientos y la ubicación de los sistemas de transmisión es competencia de entidades especializadas designadas por los Estados para controlar y regular los servicios de comunicaciones, y pueden seguir o no las pautas internacionales. Por su parte, el establecimiento de los protocolos de medición es tarea tanto de los organismos de control sanitario como de las autoridades de control de las comunicaciones y en su elaboración deben participar académicos, científicos y técnicos - tanto nacionales como extranjeros- a fin de garantizar que el sistema de control se base en valores confiables que permitan fiscalizar correctamente la intensidad de los CEM.

\section{LOS EFECTOS ASOCIADOS Y LA POLÍTICA DE CONTROL DE RIESGOS}

La necesidad de contar con una norma que establezca los valores de exposición máxima permitida se debe a los trastornos que las radiaciones no ionizantes pueden ocasionar en los organismos vivos. Según la Organización Mundial de la Salud (OMS) estos efectos se clasifican como biológicos cuando la exposición a un CEM produce alteraciones en algún sistema biológico, tales como cambios en la concentración o el transporte de alguna sustancia. Los efectos biológicos pueden sobrepasar el umbral que el cuerpo humano puede compensar y así menoscabar la salud. Estos efectos sanitarios adversos por exposición a radiofrecuencias y microondas pueden ser térmicos o atérmicos.

Los efectos térmicos son el resultado de la interacción entre un CEM y un sistema biológico, con la posterior transformación de la energía electromagnética del campo en energía térmica debido a las pérdidas dieléctricas y resistivas que sufren los tejidos biológicos. Esto provoca el incremento de la temperatura, ya sea en la zona irradiada por el CEM o en todo el organismo, en dependencia de las condiciones de exposición y de la frecuencia del CEM. Los efectos térmicos más estudiados están relacionados
FIGURA 2. Variación de la tasa de absorción específica $\left(\mathrm{SAR}^{\mathrm{a}}\right)$ promedio según la frecuencia y la zona del cuerpo irradiada

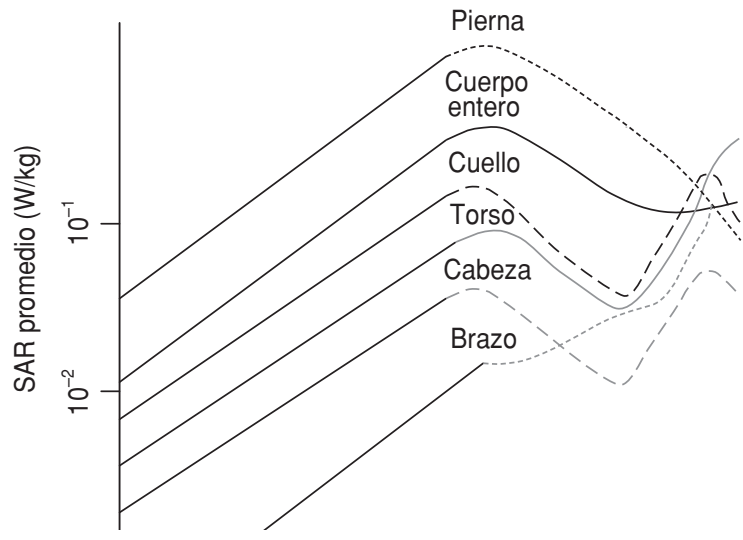

Fuente: Referencia 6. (Reproducido con permiso del Ministerio de Salud y Acción Social de la República Argentina).

a Por la sigla inglesa correspondiente a specific absorption rate.

con el deterioro o la pérdida de la visión y de la fertilidad, ya que al estar el cristalino y las gónadas en zonas de poca irrigación sanguínea, el calor generado por la acción del CEM no se disipa con facilidad.

Los efectos atérmicos se producen como resultado de la exposición a CEM de muy baja intensidad sin elevación de la temperatura en los sistemas biológicos. Si bien los efectos nocivos del efecto atérmico no se han podido corroborar mediante grandes estudios epidemiológicos, uno de los problemas de mayor actualidad relacionado con las radiaciones no ionizantes es el análisis de la exposición prolongada a la radiación de baja intensidad y su posible asociación con algunas afecciones endocrinas, malformaciones congénitas, cambios de carácter (efectos etológicos) y el cáncer.

Las normas que fijan los valores de exposición máxima permitida a las radiaciones no ionizantes de distintas frecuencias en la mayoría de los países se basan en los efectos térmicos, es decir, para cada grupo de frecuencias se fija un valor de exposición máxima permitida por debajo del cual la absorción promedio del CEM por el cuerpo humano no representará un incremento nocivo de la temperatura (en general de alrededor de $0,1^{\circ} \mathrm{C}$ ). De esta forma se pueden elaborar gráficos que ilustren el comportamiento de la tasa de absorción específica (SAR, por la sigla inglesa correspondiente a specific absorption rate) en función de la frecuencia y se pueden fijar los valores permitidos de densidad de potencia, de campo eléctrico y de campo magnético, ya sea para trabajadores (exposición a CEM durante 8 horas diarias) o para el público en general (exposición a CEM de duración indefinida) (figura 2). 
De acuerdo con los efectos mencionados anteriormente y a fin de evitar el daño que la exposición a los CEM podría provocar, en los últimos 25 años se han definido criterios y límites de exposición de carácter obligatorio que ayudan a reducir los riesgos asociados con la exposición a los CEM.

Debido a la falta de consenso acerca de las normas que se deben adoptar - y hasta que diversos estudios epidemiológicos aún no concluidos permitan establecer los criterios más racionales- se han establecido principios de cumplimento voluntario (4) que pueden contribuir a reducir al mínimo los daños ocasionados por las emisiones de radiofrecuencias.

1. Principio de precaución o de incertidumbre científica. Según este principio, se deben tomar precauciones para evitar la exposición a los CEM hasta que los conocimientos científicos y la información epidemiológica permitan definir de manera más precisa los efectos de los CEM, incluso de las emisiones de baja intensidad o de la exposición a largo plazo.

2. Principio de prudencia. Establece que se deben tomar medidas de protección de bajo costo que permitan disminuir la intensidad de los CEM.

3. Principio de exposición tan baja como sea razonablemente posible. Se debe tratar de emplear la menor potencia posible para una tarea dada. Este es un principio de precaución conocido en el campo de las radiaciones ionizantes y que se adoptó como política para el control de los riesgos por radiaciones no ionizantes.

Estos principios no establecen ni recomiendan valores máximos o mínimos, sino que enuncian conceptos que pueden interpretarse subjetivamente. Como resultado, en la actualidad se ha desatado un gran debate sobre el alcance y la aplicación de cada uno de ellos (5).

\section{LOS LÍMITES DE EXPOSICIÓN A LAS RADIACIONES NO IONIZANTES}

Al igual que para las radiaciones ionizantes, para las radiaciones no ionizantes se han establecido límites de exposición para personas expuestas en su profesión y para el público. El uso de radiofrecuencias con fines médicos se excluye de este análisis, ya que no puede establecerse un límite para los pacientes expuestos a las radiaciones - tanto para el diagnóstico como para el tratamiento- porque el beneficio que se espera es superior al posible daño ocasionado.

Para ilustrar la forma en que se regula la exposición a las radiaciones no ionizantes, se expondrá con mayor detalle la situación existente en Ar- gentina. Los límites de exposición promulgados por el Ministerio de Salud y Acción Social de ese país mediante la Resolución 202/95 se basaron en la propuesta de la Dirección Nacional de Calidad Ambiental, adscrita a la Secretaría de Salud (6-7), y aunque fueron concebidas mucho antes que los recomendados por la Comisión Internacional de Protección contra Radiaciones no Ionizantes (CIPRNI) (8), son muy parecidos.

\section{La exposición ocupacional}

El criterio empleado para determinar el límite de exposición para las personas expuestas a radiofrecuencias por razones de trabajo se basó en una jornada laboral de 40 horas semanales (con breves períodos de exposiciones elevadas) durante 50 semanas al año. Se les debe informar claramente a los trabajadores de los posibles riesgos asociados con sus ocupaciones (9). Los límites de exposición ocupacional se aplican en dependencia de la frecuencia de la exposición del cuerpo completo.

Estos límites de exposición ocupacional equivalen aproximadamente a la densidad de potencia de una onda plana incidente necesaria para producir una SAR promedio de cuerpo completo de $0,4 \mathrm{~W} / \mathrm{kg}$ (una SAR de $4 \mathrm{~W} / \mathrm{kg}$ provoca un aumento de la temperatura corporal de aproximadamente $1{ }^{\circ} \mathrm{C}$ ).

El valor mínimo de la densidad de potencia para la exposición ocupacional es de $1,0 \mathrm{~mW} / \mathrm{cm}^{2}$, lo que coincide con el espectro de frecuencias donde el cuerpo humano se comporta como una antena receptora ideal (figura 3). Como se sabe, la energía radiante se transmite y se recibe a través de elementos llamados antenas. Las configuraciones y dimensiones de las antenas dependen de diversos factores, como la frecuencia y su polarización, y en el caso más simple son irradiantes verticales (cualquier elemento conductor) con una longitud acorde con la frecuencia de emisión. Una persona de aproximadamente 1,80 metros de altura puede comportarse como una antena ideal de 0,25 longitud de onda en determinado espectro de frecuencias (por ejemplo, $40 \mathrm{MHz}$ ) y por lo tanto puede absorber con mayor eficiencia la energía irradiada.

\section{La exposición poblacional}

La población en general — que obviamente es mucho más numerosa que la población expuesta a las radiaciones por razones laborales- puede correr riesgos que por lo general no se pueden controlar individualmente. Por ello se establece que los valores límite de exposición de la población en ge- 
FIGURA 3. Valores límite para la densidad de potencia según la frecuencia (f) en Argentina ${ }^{a}$

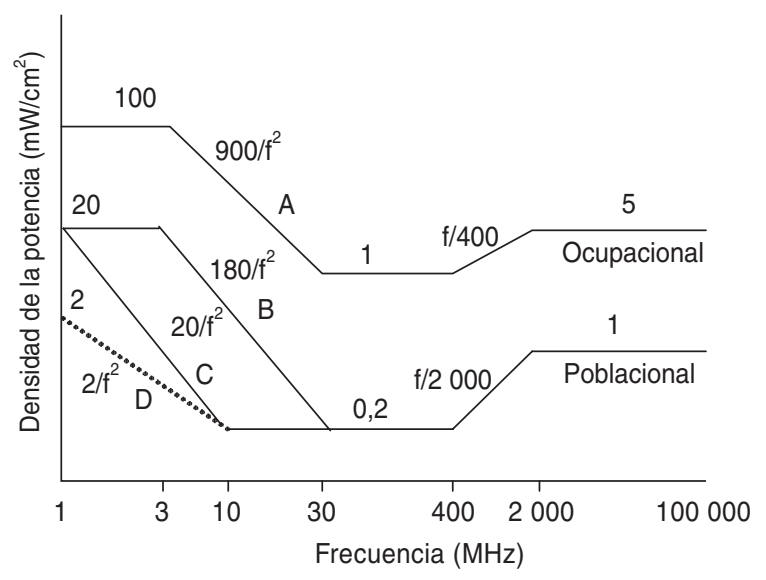

a Estas curvas reflejan el valor promedio de la densidad de potencia medida durante 6 minutos para entornos ocupacionales o durante 30 minutos para entornos poblacionales.

Leyenda:

Curva A: Representa los valores límite de exposición para entornos ocupacionales con una exposición diaria de 8 horas.

Curvas B y C: Representan los valores límite de exposición para entornos poblacionales con una exposición diaria de 24 horas, que entrarán en vigencia con el aumento planificado de nuevas fuentes radiantes, produciéndose un mayor nivel de exposición para el público. Cuando el aumento de las fuentes es significativo, se toma como referencia la curva $C$ en lugar de la $B$.

Curva D: Se aplica a entornos poblacionales cercanos a campos de antenas de frecuencia media.

(Reproducido con permiso del Ministerio de Salud y Acción Social de la República Argentina). neral deben ser la quinta parte de los valores límite aceptados para la exposición ocupacional en la mayor parte del espectro (de $10 \mathrm{MHz}$ a $300 \mathrm{GHz}$ ) y equivaler a la densidad de potencia de una onda plana incidente necesaria para producir una tasa de absorción especifica promedio de cuerpo completo de $0,08 \mathrm{~W} / \mathrm{kg}$ (figura 3).

Cuando los campos están cercanos se observa una distorsión del campo de radiofrecuencias, por lo que la exposición límite se debe determinar según la intensidad $(\mathrm{E})$ y el vector $(\mathrm{H})$ del campo magnético (figuras 4 y 5).

\section{LOS PROTOCOLOS DE MEDICIÓN}

Para que las normas que regulan la exposición máxima permitida a las radiaciones no ionizantes tengan utilidad práctica, se debe establecer una metodología de medición que permita determinar correctamente los valores de los CEM o su densidad de potencia (10).

En los centros urbanos pueden emplearse CEM de distintas frecuencias, pertenecientes a distintos servicios, por lo que es posible que en un punto dado se sienta de manera simultánea el efecto de más de una fuente. A fin de realizar una caracterización con la mayor precisión posible, a continuación se enumeran las reglas básicas que se

FIGURA 4. Valores de exposición máxima permitida para campos eléctricos en el espectro de radiofrecuencias y microondas en entornos poblacionales, según la frecuencia (f)

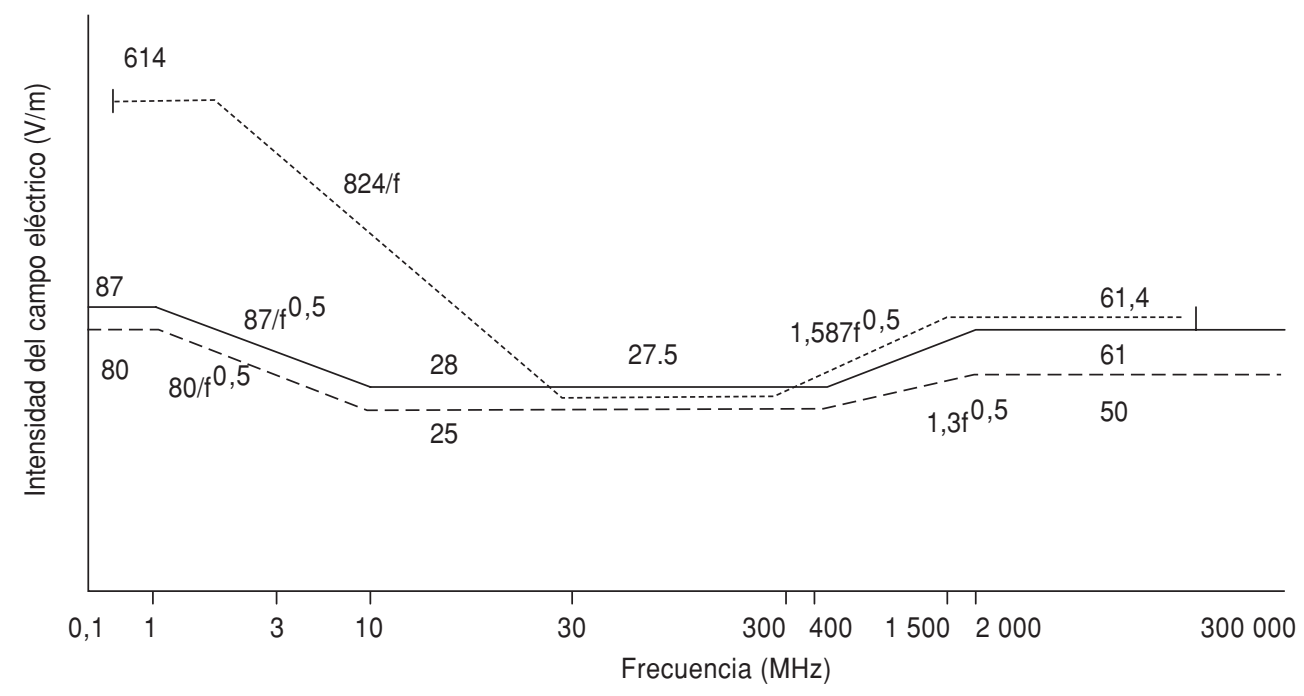

Leyenda:

........... Valores fijados por la norma de Bolivia, tomados de la Comisión Federal de Comunicaciones de los Estados Unidos de América (FCC);

_ Valores fijados por las normas de Argentina, Brasil y Perú, todos coinciden con los valores CIPRNI;

----- Valores fijados por la norma de la Republica Bolivariana de Venezuela.

(Reproducido con permiso del Ministerio de Salud y Acción Social de la República Argentina). 
FIGURA 5. Valores de exposición máxima permitida para campos magnéticos en el espectro de radiofrecuencias y microondas en entornos poblacionales según la frecuencia (f)

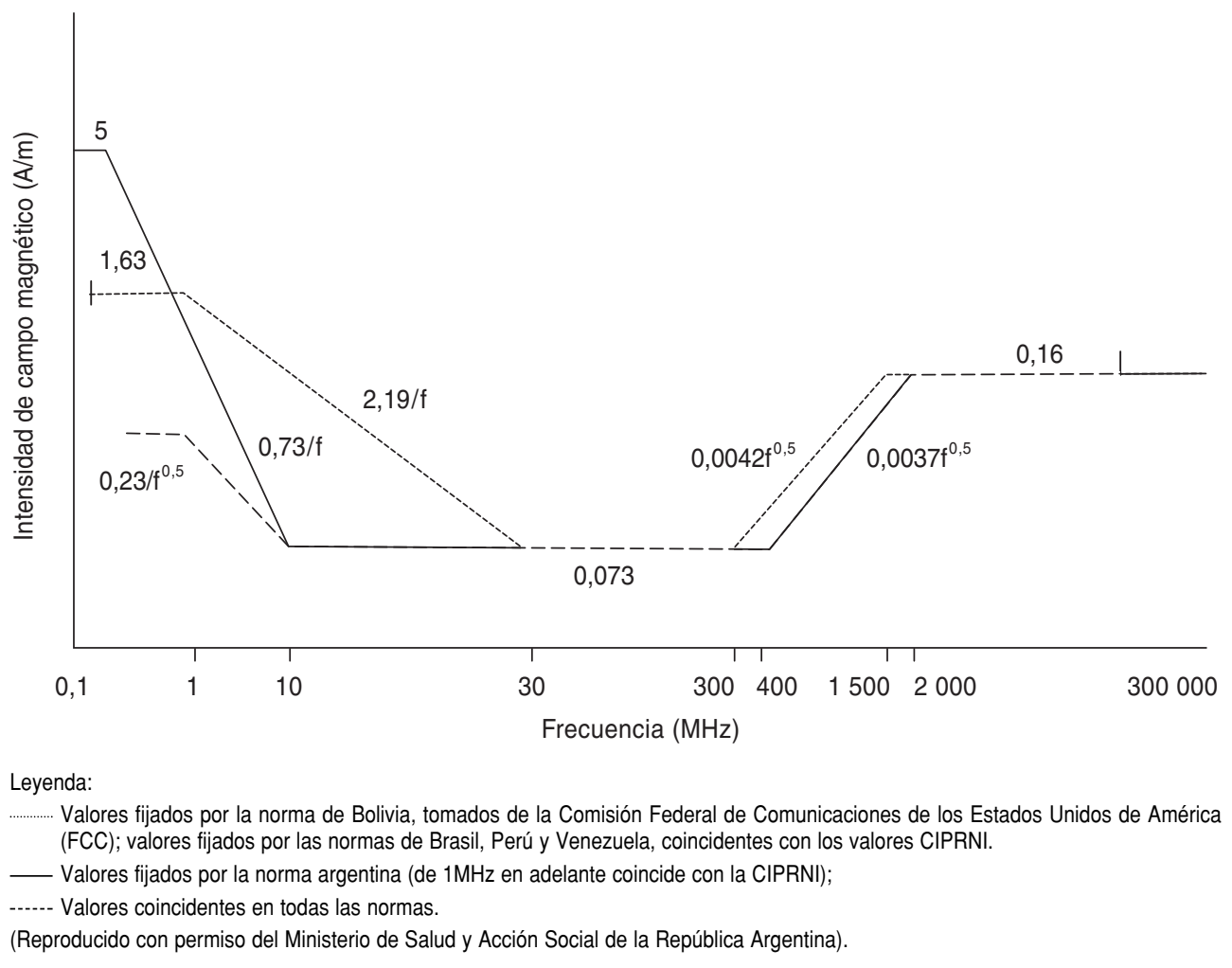

deben tener en cuenta para medir correctamente los parámetros de los CEM.

Por lo general, los puntos de medición en los centros urbanos no suelen ser zonas abiertas en las que los CEM se reciben de forma directa, sino puntos donde el CEM resultante se construye mediante la suma vectorial de los múltiples efectos que provocan los obstáculos circundantes. Ya sea en lugares cerrados - como un hospital, un colegio o una vivienda - o en zonas de tránsito - como una calle de la ciudad o un parque-, el punto de medición se ve afectado por diversos CEM provenientes de distintas fuentes conocidas previamente o no.

La medición del valor individual de los campos eléctrico y magnético, o de la densidad de potencia asociada con el CEM se puede realizar mediante la integración por banda ancha o por banda angosta.

La integración por banda ancha se realiza mediante monitores de radiación con sondas isotrópicas sensibles a los campos eléctricos o magnéticos, en dependencia de la magnitud que se desee medir. Estos equipos ofrecen el valor resultante de la in- tegración de todos los componentes del campo (eléctrico o magnético, según el caso) en las tres direcciones principales y captan todas las emisiones provenientes de los distintos servicios y fuentes, aunque no pueden discriminarlas. Por tanto, el método de integración por banda ancha solo permite obtener un valor total de los campos actuantes, sin una discriminación espectral.

Cuando es necesario identificar las fuentes que emiten radiaciones en un punto dado, se debe utilizar el método de integración por banda angosta. Esta técnica utiliza un analizador de espectro capaz de integrar la potencia de las emisiones, pero cuenta además con un conjuntos de antenas de parámetros conocidos, calibradas en el entorno espectral de trabajo, que le permite caracterizar cada una de las fuentes emisoras, aunque sean de distintos servicios. De esta forma se puede saber qué componente del campo (eléctrico o magnético, en dependencia de la antena utilizada) aporta cada una de las emisiones.

Una vez escogido el método de medición apropiado, se debe prestar atención al tipo de tarea o actividad que se realiza en el punto que se desea 
caracterizar. Si está ubicado dentro de una institución dedicada a la salud o a la educación, es estrictamente necesario realizar la medición en el lugar donde se encuentra el paciente o el alumno, según el caso, pues se deben medir los campos a la altura en que la persona realiza sus tareas. Estas particularidades deben mencionarse explícitamente en el protocolo de medición.

Además, se debe establecer que solo se utilicen instrumentos calibrados y respaldados con certificados de validez internacional, de manera que las mediciones presenten un grado de exactitud adecuado, independientemente del organismo o del profesional que las realice. Esto permitirá comparar los valores con los de otros estudios y perfeccionar la técnica.

\section{NORMAS ESTABLECIDAS EN AMÉRICA LATINA}

Solo diez países latinoamericanos poseen normas que regulan las dosis de exposición permitida a las radiaciones no ionizantes. Algunos establecieron los valores límite según las recomendaciones del Instituto Nacional de Normas de los Estados Unidos de América (American National Standards Institute, ANSI) aprobadas en 1974 por su Comité C-95. En 1991, el ANSI recomendó su nuevo estándar C-95.1-1991, en el que estableció el límite de exposición ocupacional de $1 \mathrm{~mW} / \mathrm{cm}^{2}$ en el espectro de frecuencias de 30 a $300 \mathrm{MHz}$. Solamente Bolivia adoptó el estándar del ANSI de 1991, basado en límites de la Comisión Federal de Comunicaciones de los Estados Unidos de América (Federal Communications Commission, FCC) (11), mientras que los otros países establecieron normas basadas en las recomendaciones del CIPRNI de 1998 (12). Algunas normas, como la de Chile, no tienen representación en frecuencias y solo fijan valores puntuales. A continuación se resume la información disponible.

Argentina. Resoluciones del Ministerio de Salud, MS 202/1995, y de la Secretaría de Comercio, SeCom 530/2000. Los límites ocupacionales y públicos son similares a los de las normas de la CIPRNI y se presentan en las figuras 2 y 3 .

Bolivia: Estándar Técnico de la Superintendencia de Telecomunicaciones, SITTEL 2002/0313.

Brasil: Resolución 303 del 2 de julio de 2002 de la Agencia Nacional de Telecomunicaciones (Agência Nacional de Telecomunicações, ANATEL) que regula los límites de exposición a campos eléctricos, magnéticos y electromagnéticos en el espectro de radio- frecuencias entre $9 \mathrm{kHz}$ y $300 \mathrm{GHz}$. Se basa en los límites recomendados por la CIPRNI.

Chile: Decreto 594/00 Salud, Título 4, sobre la contaminación ambiental y Resolución 505/00 de la Subsecretaría de Telecomunicaciones, SUBTEL.

Colombia: Norma Técnica UIT K52, basada en los límites recomendados por la CIPRNI.

Costa Rica: Resolución No 2896-98 de la Sala Constitucional que establece protocolos de medición para las líneas de alta tensión.

Ecuador: Norma Técnica que establece los límites de máxima exposición permitida, aprobada en 2004. Se basa en los límites recomendados por la CIPRNI.

México: La Comisión Federal de Telecomunicaciones de México, COFETEL, reitera en su Programa Nacional de Normalización 2005 (PNN-2005) la necesidad de aprobar una norma oficial mexicana (NOM) que regule las radiaciones no ionizantes en todo el espectro radioeléctrico. Este reclamo, planteado hace varios años en la NOM-126, refleja la preocupación social expresada por sectores cada vez más amplios de la población.

Perú: Decreto Supremo del Ministerio de Transportes y Comunicaciones, MTC 038-2003, sobre la adopción de límites de exposición en el espectro de radiofrecuencias de $9 \mathrm{kHz}$ a $300 \mathrm{GHz}$. Se basa en los límites recomendados por la CIPRNI.

Venezuela: Norma del Comité Venezolano para Normas Industriales, COVENIN: Norma Venezolana Covenin, NVC 2238-00. Es una norma nacional que fija los límites de máxima exposición permitida.

Como se puede observar, los países que han aprobado recientemente normas con los límites de exposición máxima permitida se han basado en las recomendaciones del ICNIRP o de la FCC.

Como conclusión, se puede afirmar que las normas y estándares empleados en algunos países de la Región se deben actualizar, completar y armonizar, de manera que abarquen todo el espectro de radiofrecuencias. Los países que aún no cuentan con este tipo de normas o están en el proceso de modernizarlas pueden utilizar como marco general la información actualizada y armonizada que brinda la Organización Mundial de la Salud mediante su proyecto internacional sobre CEM, que se basa en los principios de precaución, prudencia y exposición tan baja como sea razonablemente posible. 


\section{SYNOPSIS}

\section{Norms and standards for radiofrequency electromagnetic fields in Latin America: guidelines for exposure limits and measurement protocols}

New technologies that use electromagnetic fields (EMF) have proved greatly beneficial to humankind. EMF are used in a variety of ways in the transmission of electrical energy and in telecommunications, industry, and medicine. However, some studies have shown that EMF could be detrimental to one's health, having found an association between exposure to EMF on the one hand, and the incidence of some types of cancer as well as behavioral changes on the other. Although so far there is no concrete proof that exposure to low-intensity EMF is hazardous, researchers continue to study the issue in an attempt to reach a consensus opinion and to establish safety standards. While developing and establishing such norms and standards have traditionally been the responsibility of international specialized agencies, national health authorities should take an active part in this process. Currently the Pan American Health Organization is promoting scientific research, often in the form of epidemiologic studies, in order to propose uniform norms and standards. Some Latin American countries, including Argentina, Brazil, Chile, Colombia, Costa Rica, Ecuador, Mexico, Peru, and Venezuela, have already enacted incomplete or partial legislation based on recommended international standards. This article describes the norms established in Latin America and the particular approach taken by each country.

Key words: environmental quality standards, electromagnetic fields, radiation exposure, Latin America.

\section{REFERENCIAS}

1. United States of America, National Institute of Environmental Health Sciences, U.S. Department of Energy. Preguntas y respuestas sobre los CEM: campos eléctricos y magnéticos asociados con el uso de la energía eléctrica. Washington, D.C.: U.S. Department of Energy; 1995.

2. United States of America, Federal Communications Comission, Office of Engineering and Technology. Evaluating compliance with FCC Guidelines for Human Exposure to Radiofrequency Electromagnetic Fields. Washington, D.C.: Federal Communications Comission; 1997. (OET Bulletin No. 65)

3. Portela A. Fundamental and applied aspects of non-ionizing radiation. New York: Plenum Press; 1974.

4. World Health Organization. Establishing a dialogue on risk from electromagnetic fields. Radiation and environmental health. Geneva: World Health Organization; 2002.

5. Foster $\mathrm{KH}$, Vecchia $\mathrm{P}$, Repacholi $\mathrm{MH}$. Science and the precautionary policy. Science. 2000; 288:979-81.
6. Portela A, Skvarca J, Matute Bravo E, Loureiro L. Volumen I: Manual de estándares de seguridad para la exposición a radiofrecuencias comprendidas entre $100 \mathrm{kHz}$ y $300 \mathrm{GHz}$. En: Prospección de radiación electromagnética ambiental no ionizante. Buenos Aires: Secretaría de Salud; 1988.

7. Portela A, Skvarca J, Matute Bravo E, Loureiro L. Volumen II: Radiación de radiofrecuencias: consideraciones biofísicas, biomédicas y criterios para el establecimiento de estándares de exposición. En: Prospección de radiación electromagnética ambiental no ionizante. Buenos Aires: Secretaría de Salud; 1988.

8. Repacholi MH. IRPA guidelines on protection against non-ionizing radiation. New York: Pergamon Press; 1991.

9. United States of America, Institute of Electrical and Electronics Engineers. IEEE recommended practice for radio frequency safety programs, $3 \mathrm{kHz}$ to 300 GHz. New York: IEEE; 2006. (IEEE Std C95.7).
10. United States of America, Institute of Electrical and Electronics Engineers. IEEE recommended practice for measurements and computations of radio frequency electromagnetic fields with respect to human exposure to such fields, $100 \mathrm{kHz}-300 \mathrm{GHz}$. New York: IEEE; 2006. (IEEE Std C95.3).

11. United States of America, Institute of Electrical and Electronics Engineers. IEEE standard for safety levels with respect to human exposure to radio frequency electromagnetic fields, $3 \mathrm{kHz}$ to $300 \mathrm{GHz}$. New York: IEEE; 2006. (IEEE Std C95.1).

12. Comisión Internacional para la Protección de las Radiaciones no Ionizantes. Guidelines for limiting exposure to time-varying electric, magnetic, and electromagnetic fields (up to $300 \mathrm{GHz}$ ) Health Physics. 1988;74(4):494-522.

Manuscrito recibido el 14 de diciembre de 2004 Aceptado para publicación, tras revisión, el 9 de marzo de 2005. 